\title{
MODELAGEM DE UM SISTEMA DE INFORMAÇÃO PARA RASTREABILIDADE NA INDÚSTRIA DO VINHO BASEADO EM UMA ARQUITETURA ORIENTADA A SERVIÇOS
}

\author{
OSVALDO GOGLIANO SOBRINHO ${ }^{1}$, CARLOS E. CUGNASCA ${ }^{2}$, FLAVIO B. FIALHO ${ }^{3}$, \\ CELITO C. GUERRA ${ }^{4}$
}

RESUMO: Apresenta-se neste trabalho pesquisa científica que levou à modelagem de um sistema de informação para o registro de dados de rastreabilidade destinado à indústria do vinho, segundo os conceitos de uma arquitetura computacional orientada a serviços (service oriented architecture, SOA). Desde 2005, a manutenção de tais registros é obrigatória para todos os produtores que pretendem exportar para países da União Europeia. Ademais, os consumidores finais, inclusive brasileiros, preocupados com a segurança alimentar, apresentam demanda crescente por informações. Buscou-se uma solução que contemple a indústria coletivamente, por meio de consórcios ou associações de produtores, diluindo custos e compartilhando benefícios. A partir do levantamento bibliográfico realizado e de contatos com pesquisadores e representantes do setor produtivo vinícola brasileiro, em Bento Gonçalves - RS, pesquisaram-se tópicos de tecnologia da informação ligados ao tema, além de detalhes sobre o processo produtivo do vinho. A partir de um modelo do processo produtivo do vinho sugerido pelos autores, empregou-se a Unified Modeling Language (UML), para a modelagem do sistema, que utiliza a tecnologia de web services para o tráfego de dados. Com um protótipo funcional, constataram-se a viabilidade do modelo e a possibilidade de sua utilização em outros domínios.

PALAVRAS-CHAVE: segurança alimentar, tecnologia da informação, internet, web services, UML.

\section{MODELING OF AN INFORMATION SYSTEM FOR WINE TRACEABILITY BASED ON A SERVICE ORIENTED ARCHITECTURE}

\begin{abstract}
The purpose is to present a scientific research that led to the modeling of an information system which aimed at the maintenance of traceability data in the Brazilian wine industry, according to the principles of a service-oriented architecture (SOA). Since 2005, traceability data maintenance is an obligation for all producers that intend to export to any European Union country. Also, final customers, including the Brazilian ones, have been asking for information about food products. A solution that collectively contemplated the industry was sought in order to permit that producer consortiums of associations could share the costs and benefits of such a solution. Following an extensive bibliographic review, a series of interviews conducted with Brazilian researchers and wine producers in Bento Gonçalves - RS, Brazil, elucidated many aspects associated with the wine production process. Information technology issues related to the theme were also researched. The software was modeled with the Unified Modeling Language (UML) and uses web services for data exchange. A model for the wine production process was also proposed. A functional prototype showed that the adopted model is able to fulfill the demands of wine producers. The good results obtained lead us to consider the use of this model in other domains.
\end{abstract}

KEYWORDS: food safety, information technology, internet, web services, UML.

\footnotetext{
${ }^{1}$ Eng $^{\mathrm{o}}$ Civil, Doutorando em Engenharia Elétrica, Laboratório de Automação Agrícola, LAA, Escola Politécnica da USP, São Paulo SP, ogogli@abili.com.br

${ }^{2}$ Eng $^{\mathrm{o}}$ Eletricista, Livre Docente, Prof. Associado, Laboratório de Automação Agrícola, LAA, Escola Politécnica da USP, São Paulo - SP.

${ }^{3}$ Eng $^{\text {o }}$ Agrônomo, Doutor, Pesquisador III, Empresa Brasileira de Pesquisa Agropecuária, Embrapa Uva e Vinho, Bento Gonçalves RS.

${ }^{4}$ Eng $^{\mathrm{O}}$ Agrônomo, Doutor, Pesquisador III, Empresa Brasileira de Pesquisa Agropecuária, Embrapa Uva e Vinho, Bento Gonçalves RS.

Recebido pelo Conselho Editorial em: 5-11-2008

Aprovado pelo Conselho Editorial em: 12-12-2009
}

Eng. Agríc., Jaboticabal, v.30, n.1, p.100-109, jan./fev. 2010 


\section{INTRODUÇÃO}

A preocupação com a questão da rastreabilidade nas cadeias alimentares ganhou destaque ao longo da década de 1990, principalmente após a série de incidentes envolvendo o possível consumo de carne bovina proveniente de animais atingidos pela encefalopatia espongiforme bovina - o mal da vaca louca - que pode desencadear em seres humanos a Síndrome de Creutzfeldt-Jakob, doença neurológica degenarativa, com consequências fatais. Essa possibilidade levou os consumidores, particularmente os europeus, a adotarem profundas mudanças em seus hábitos alimentares (McEACHERN \& SEAMAN, 2005).

Tais fatos levaram o PARLAMENTO EUROPEU E CONSELHO DA UNIÃO EUROPEIA (2002) à promulgação do Regulamento (CE) 178/2002, que tornou obrigatória, a partir de janeiro de 2005, a manutenção de dados de rastreabilidade para todos os envolvidos na comercialização de produtos alimentícios em qualquer país da União Europeia.

Já que a legislação europeia considera o vinho como produto alimentício, os produtores brasileiros que pretendem exportar para qualquer país da União Europeia, estão obrigados a adotar práticas de rastreabilidade para seus produtos. Curiosamente, a legislação brasileira considera o vinho bebida alcoólica, contrariamente à pretensão da indústria brasileira, já que sua classificação como produto alimentício levaria à redução da carga tributária incidente sobre o produto.

Alguns podem considerar exagerada a inclusão do vinho no escopo do Regulamento 178/2002. Embora não tão graves como os episódios relacionados ao consumo de carne, incidentes de segurança alimentar envolvendo o consumo de vinho têm sido relatados, desde a introdução acidental de partículas de vidro no interior das garrafas, até a contaminação acidental por substâncias nocivas.

A busca por produtos garantidos e de melhor qualidade ocorre também no Brasil. Segundo relato de ROMBALDI et al. (2007), a "origem conhecida e a garantia de qualidade" estão entre os cinco quesitos mais citados por consumidores de frutas no Estado do Rio Grande do Sul. Segundo o mesmo trabalho, há duas décadas esses quesitos não eram sequer mencionados. Dessa forma, se além de cumprirem exigências legais, os produtores vinícolas brasileiros forem capazes de atender às demandas dos consumidores sobre rastreabilidade e informação sobre qualidade, estarão agregando significativo valor a seus produtos (GOGLIANO SOBRINHO \& CUGNASCA, 2005).

Para os produtores, resumidamente, o Regulamento (CE) 178/2002 exige que qualquer unidade ou lote de produtos seja identificada por código e que seja possível informar todos os insumos e seus fornecedores utilizados em qualquer unidade do produto ou lote, assim como todos os seus compradores.

No Brasil, a norma NBR ISO 22000 (ABNT, 2006) também trata da rastreabilidade aplicada a organizações produtoras de alimentos. Encontra-se em seu item 7.9: "A organização deve estabelecer e aplicar um sistema de rastreabilidade que permita a identificação de lotes de produtos e sua relação com lotes de matérias-primas, processamento e registros de liberação...".

A utilização de soluções que atendem a associações ou consórcios de produtores vinícolas já em operação na Europa, traz substanciais vantagens para os produtores associados. Por exemplo, o CONSORZIO DEL VINO BRUNELLO DI MONTALCINO (2006), na Itália, disponibiliza em seu portal Internet dados qualitativos de cada unidade de seus produtos, a partir do código único da garrafa. A adoção de um modelo adequado à utilização coletiva foi um dos requisitos para o sistema modelado nesta pesquisa.

\section{MATERIAL E MÉTODOS}

A pesquisa teve início com uma extensa revisão bibliográfica. Diversos temas precisaram ser abordados: o conceito de rastreabilidade nas cadeias alimentares e os fatos que a tornaram tão importante ao longo dos últimos anos; a importância da rastreabilidade no marketing de produto, inclusive a concessão das denominações geográficas; o processo produtivo do vinho; aspectos 
legais relacionados à rastreabilidade; incidentes de segurança alimentar envolvendo o consumo de vinho; todos os aspectos de tecnologia de informação relacionados ao tema.

A partir da revisão da literatura, que marcou o início da pesquisa, a sequência do trabalho passou pelas seguintes etapas: visitas e reuniões técnicas com representantes do setor produtivo brasileiro e com pesquisadores da Embrapa Uva e Vinho, na cidade de Bento Gonçalves - RS, por meio dos recursos concedidos pela Fundação de Amparo à Pesquisa do Estado de São Paulo (Fapesp), por meio de Auxílio à Pesquisa; proposição de modelo para o processo produtivo do vinho; modelagem do sistema de informação segundo os requisitos de uma arquitetura orientada a serviços (usualmente representada pela sigla SOA, do inglês service oriented architecture); construção de protótipo funcional para testes, e avaliação de resultados.

Efetuaram-se cinco viagens a Bento Gonçalves, a partir de 2005. Participaram das reuniões técnicas, representantes e consultores técnicos de vinícolas (Aliança, Aurora, Miolo e Salton), pesquisadores da Embrapa Uva e Vinho, também sediada em Bento Gonçalves, representantes e consultores técnicos de entidades de classe (Instituto Brasileiro do Vinho, Ibravin e Associação dos Produtores do Vale dos Vinhedos, Aprovale).

Os contatos com representantes do Ibravin, Aprovale e Embrapa Uva e Vinho reforçaram a intenção de procurar uma solução adequada à utilização coletiva.

As viagens também permitiram visitas a instalações produtivas de vinícolas (Aurora, Casa Valduga, Miolo e Salton) e às sedes do Ibravin e da Embrapa Uva e Vinho. Nesses contatos, as diversas etapas envolvidas no processo produtivo do vinho foram detalhadas.

Durante esses contatos, discutiu-se a questão da rastreabilidade junto à indústria vinícola, apresentou-se a ideia de um sistema de informação para rastreabilidade com utilização coletiva, suas vantagens e características, discutiram-se detalhes sobre o processo produtivo do vinho; discutiram-se requisitos do sistema.

Em seu Glossário de Web Services (WORLD WIDE WEB CONSORTIUM, 2007), o W3C refere-se à arquitetura orientada a serviços como "um conjunto de componentes que podem ser invocados e cujas descrições de interfaces podem ser publicadas e descobertas". Sua utilização na operação conjunta de sistemas na indústria é crescente (JARDIM-GONÇALVES et al., 2006). A necessidade de integração entre os sistemas de informação dos diversos participantes de uma cadeia produtiva é mais um incentivo para a adoção dessa ideia. Modelos para essa integração têm sido sugeridos, como o apresentado no trabalho de BECHINI et al. (2008).

A modelagem do sistema, segundo os preceitos de uma arquitetura SOA, foi motivada por sua utilização em um contexto coletivo, na qual a interação com sistemas de informação dos vários participantes é fundamental.

A modelagem do sistema foi efetuada por meio da Unified Modeling Language (UML), e um protótipo funcional foi construído para a avaliação de resultados. O protótipo funcional foi instalado em um servidor web operando com sistema operacional FreeBSD 6.2 e Apache 2.2.6. Como Sistema Gerenciador de Banco de Dados, utilizou-se o MySQL 5.0.45 e como linguagem de programação foi utilizado o PHP 5.2.4. Todas essas opções foram feitas considerando-se sua larga utilização, sua característica de software aberto e sua disponibilidade em um servidor web especialmente configurado para esses testes. A opção por ferramentas de software aberto justificase também pelo aspecto da necessária operação integrada a sistemas desenvolvidos em diversas plataformas e aos riscos inerentes a uma escolha de ferramentas comerciais quando o desenvolvimento dessas é abandonado pela empresa produtora (HENLEY \& KEMP, 2008). O sistema fornece serviços na forma de web services utilizando o protocolo SOAP (WORLD WIDE WEB CONSORTIUM, 2007). Buscou-se seguir a metodologia da espiral evolucionária apresentada por BOEHM (1988).

\section{RESULTADOS E DISCUSSÃO}

Apresenta-se, na Figura 1, uma visão simplificada da arquitetura proposta para o sistema. 


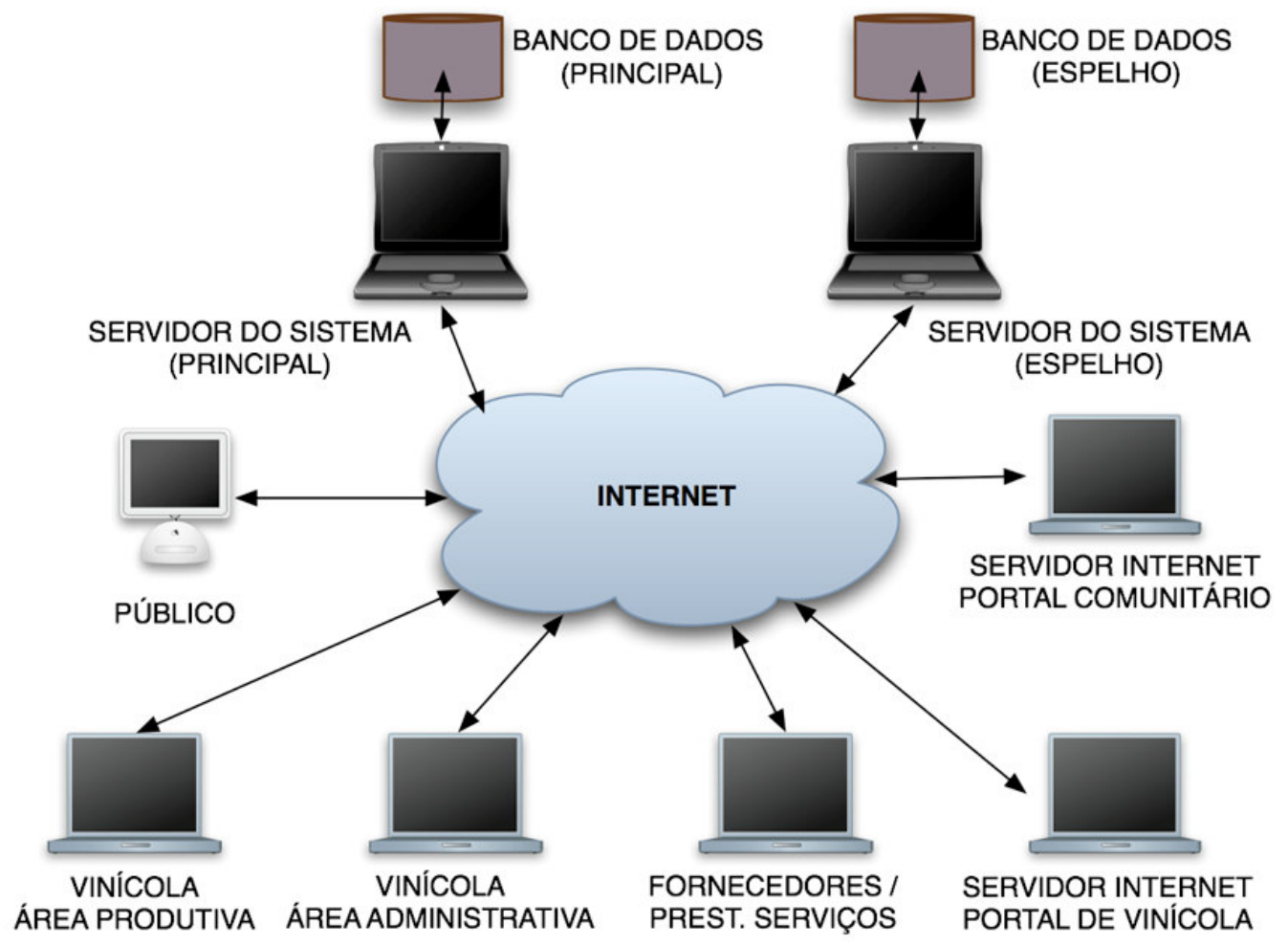

FIGURA 1. Arquitetura simplificada. Simplified architecture.

Fisicamente, o sistema e seu sistema gerenciador de banco de dados poderão ser instalados com espelhamento em entidades de referência, como associações de produtores, universidades, etc.

Nesse contexto, o sistema atua como fornecedor de serviços, interagindo com sistemas externos. Por exemplo, sistemas de controle de produção instalados em vinícolas poderão enviar informações sobre rastreabilidade obtidas durante a evolução do processo produtivo; os sistemas administrativos nas vinícolas poderão obter dados de rastreabilidade na forma de serviços fornecidos pelo sistema modelado; portais internet de vinícolas ou associações poderão fornecer diretamente ao público consumidor informações sobre determinadas garrafas de vinho, obtendo essas informações por meio de serviços junto ao sistema modelado; pequenas vinícolas poderão utilizar sistemas simplificados, baseados em web, para a inserção de dados de sua produção; fornecedores e prestadores de serviços aos produtores poderão interagir com o sistema por meio dos serviços oferecidos.

A modelagem do processo produtivo do vinho foi efetuada a partir de conceito apresentado por NATALE et al. (2002). Nesse modelo, o processo produtivo do vinho é considerado como uma sequência de etapas. Cada etapa tem início quando um contêiner recebe o conteúdo gerado por etapas anteriores. A etapa termina quando esse conteúdo é transferido para outros contêineres. Durante uma etapa, insumos podem ser adicionados, processos industriais podem ser aplicados e amostras podem ser colhidas para análises qualitativas. Esquematicamente, apresenta-se esse conceito na Figura 2.

No decorrer de uma etapa, dada a homogeneidade do volume armazenado no contêiner, seu histórico de rastreabilidade é único.

A partir das ideias expostas no trabalho previamente referenciado e no conceito de etapa de produção exposto anteriormente, desenvolveu-se o diagrama Entidade-Relacionamento adotado na modelagem dos dados, apresentado na Figura 3. 


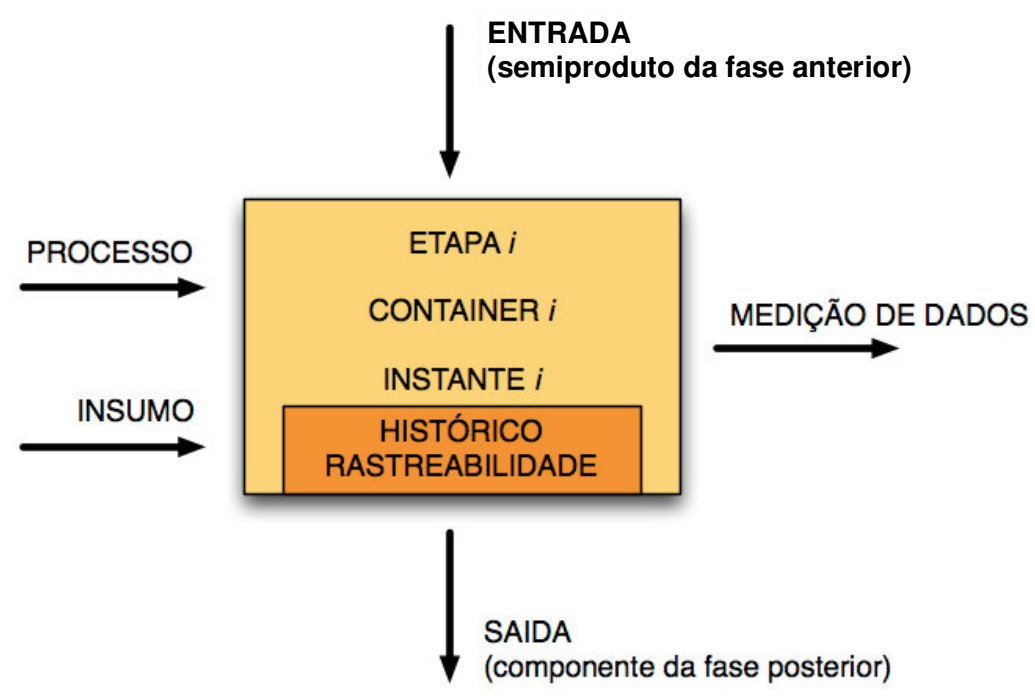

FIGURA 2. Etapa do processo produtivo do vinho. Wine production stage.

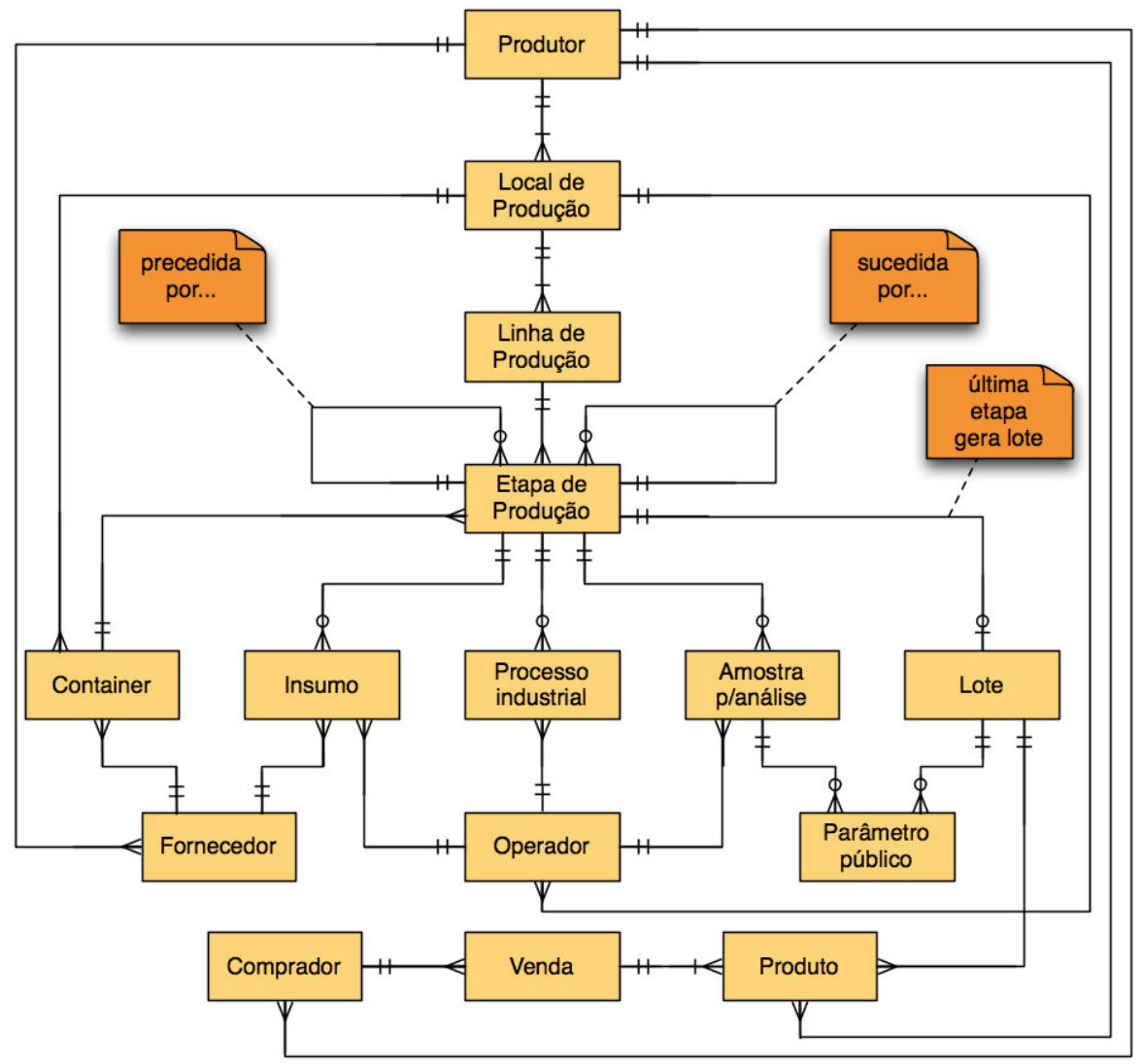

FIGURA 3. Diagrama entidade-relacionamento. Entity-relationship diagram.

A seguir, comentam-se as diversas entidades envolvidas:

Produtor: é a pessoa física ou jurídica que produz vinho.

Local de produção: também conhecido como cantina, é o local onde ocorre a produção do vinho.

Linha de produção: caracteriza um conjunto interconectado de etapas de produção, tendo início no recebimento das uvas, e prosseguindo até o engarrafamento e a expedição do produto. 
Etapa de produção: caracteriza-se pela manutenção do produto em um único local de armazenamento, o contêiner. Cada etapa é precedida por zero (primeira etapa) ou mais etapas. Também é sucedida por zero (etapa final) ou mais etapas. Esse encadeamento forma uma rede que caracteriza toda a linha de produção.

Contêiner: local onde ocorre uma etapa da produção de vinho, usualmente um tanque de grande capacidade. Os contêineres podem ser individuais (por exemplo, um tanque de grande capacidade) ou um grupo (por exemplo, um lote de barris de madeira de pequena capacidade). No conceito desta modelagem, pode-se considerar um grupo de contêineres como sendo um único contêiner, sempre que o conteúdo de todo o grupo for homogêneo.

Insumo: produto industrial, vegetal, mineral ou de ação biológica adicionado aos contêineres durante as etapas de produção do vinho.

Processo industrial: qualquer processo industrial aplicado ao conteúdo de um contêiner durante uma etapa do processo.

Amostra para análise: cada etapa permite a coleta de zero ou mais amostras do produto para análises laboratoriais ou de outra ordem.

Operador: pessoa física encarregada da adição de insumos, aplicação de um processo industrial ou coleta de amostras para análise durante as etapas de produção.

Fornecedor: pessoa física ou jurídica que fornece insumos ou contêineres utilizados nas etapas produtivas.

Lote: cada etapa final de uma linha de produção dá origem a um lote formado por garrafas de vinho, caixas de garrafas e paletes.

Parâmetro público: cada análise laboratorial pode dar origem a um parâmetro indicativo de qualidade do produto. Esses parâmetros estão relacionados a uma etapa do processo produtivo e a um lote. Poderão ser fornecidos pelo sistema de informação para a utilização, por exemplo, em portais Internet do produtor ou de uma associação de produtores, correlacionando garrafas individuais com o valor do parâmetro colhido.

Produto: unidade do produto pronta para ser comercializada. Pode apresentar-se na forma de garrafas, caixas de garrafas ou paletes contendo diversas caixas.

Comprador: pessoa física ou jurídica que adquire produtos por meio de um processo de venda.

Venda: relaciona produtos e compradores.

A modelagem do sistema proposto passa pela definição das diversas classes ligadas às entidades descritas. Apresenta-se, na Figura 4, a visão geral de todas as classes e suas associações. 


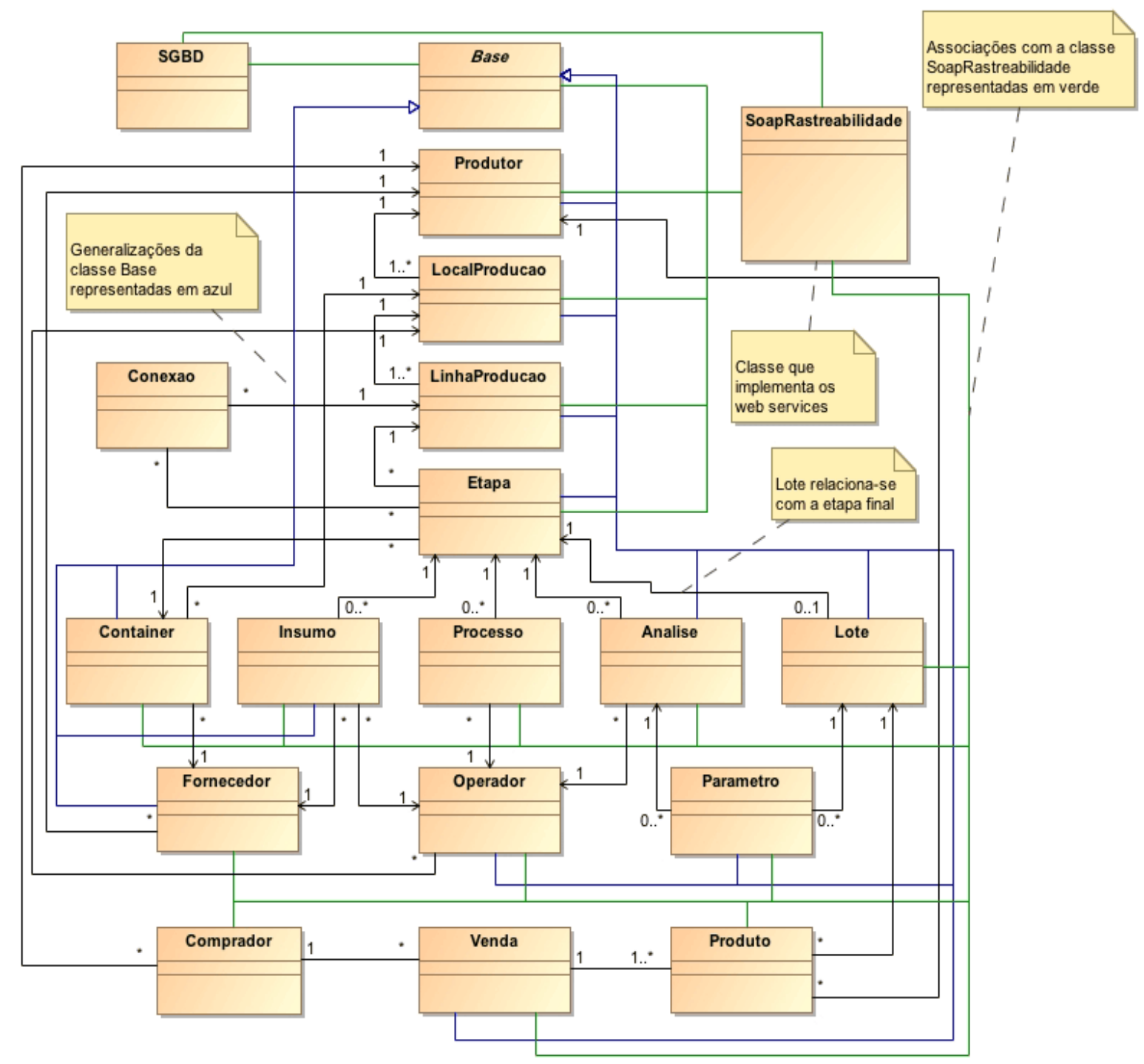

FIGURA 4. Diagrama de classes. Class diagram.

Observa-se que, praticamente, todas as classes representam as entidades exibidas na Figura 3. Além dessas, introduzem-se outras:

SGBD: implementa as propriedades e as operações necessárias para a interação com o SGBD.

Conexão: implementa as propriedades e as operações necessárias para conectar as etapas de produção do vinho.

Base: praticamente todas as classes representadas são generalizações dessa classe, que possui duas propriedades fundamentais: chave - código único que identifica as diversas instâncias dos objetos dessa classe e de suas subclasses, ou seja, praticamente todos os objetos instanciados no sistema. Na implementação do protótipo funcional, utilizou-se como código de uma sequência de 32 caracteres aleatórios gerados por uma função hash; sgbd - instância do objeto SGBD, responsável por todas as interações dos diversos objetos com o sistema de gerenciamento de banco de dados.

SoapRastreabilidade: implementa as 74 operações que são oferecidas como web services.

Apresenta-se o diagrama de implantação do protótipo funcional na Figura 5. 


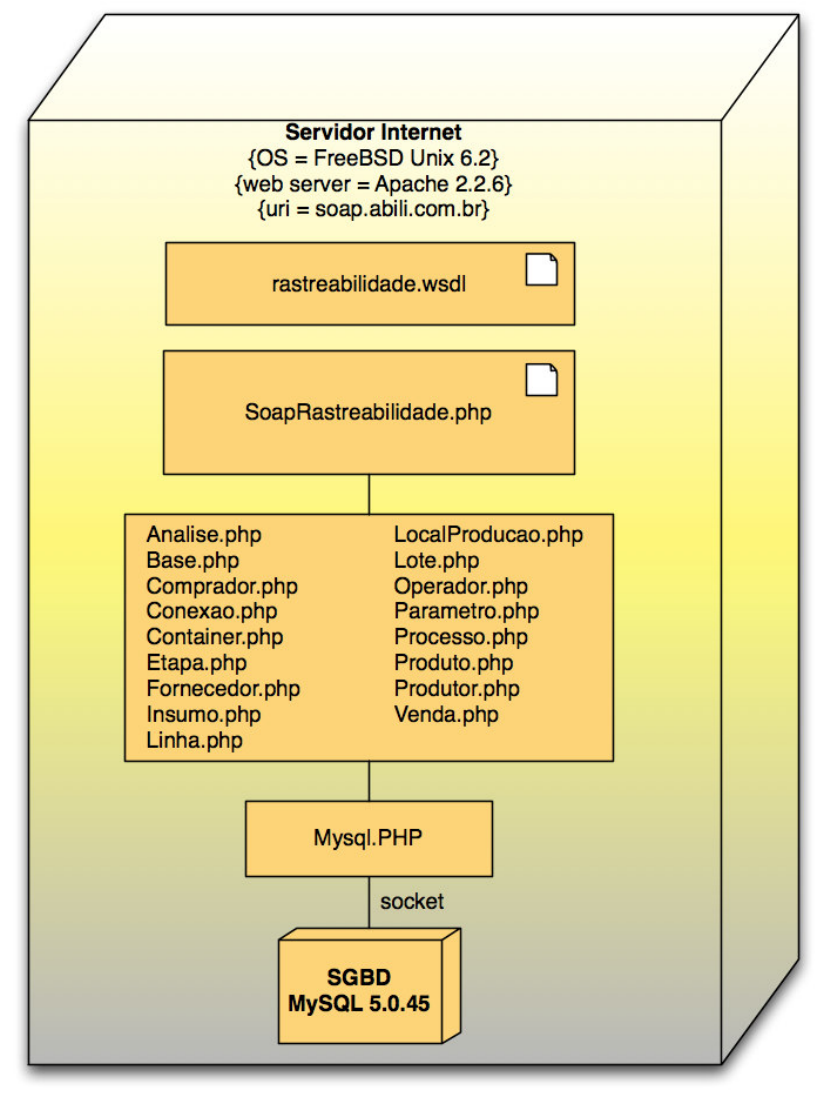

FIGURA 5. Diagrama de implantação. Deployment diagram.

Quanto à verificação de atendimento a requisitos, embora esse seja um longo processo que demanda intenso trabalho de utilização e verificação sistemática de resultados, durante os testes efetuados com o protótipo funcional, não se encontrou nenhuma falha na modelagem de dados e na modelagem do sistema e relacionamento de classes que pudessem comprometer um futuro desenvolvimento de um sistema de informação baseado no modelo proposto.

Por último, uma possibilidade interessante a ser verificada é a utilização do sistema modelado junto a outros domínios. Parece razoável supor que o sistema de rastreabilidade aqui proposto possa ser aplicado a produtos como líquidos, pós, grãos e assemelhados. No caso do vinho, uma restrição decorre do fato de que nunca um contêiner fica parcialmente cheio, pois essa situação levaria à oxidação e perda do produto. No entanto, isto pode ocorrer com outros tipos de produtos, e as consequências desse fato podem levar à necessidade de ajustes no modelo do processo produtivo e/ou na modelagem do sistema de informação.

\section{CONCLUSÕES}

O sistema modelado atende às três exigências básicas do Regulamento (CE) 178/2002 - a capacidade de informar, por unidade de produto, todos os compradores, os insumos empregados e seus fornecedores.

Quanto a funcionalidades ligadas ao marketing do vinho, o sistema permite o registro e a divulgação de parâmetros públicos de qualidade, por unidade produzida, de maneira análoga ao que se encontra atualmente disponível em portais Internet de associações de produtores vinícolas europeus. O registro de dados geográficos, como latitude e longitude de fornecedores e produtores, permite a interação com serviços de mapeamento on-line, como, por exemplo, o Google Maps. Esse tipo de interação auxilia em ações ligadas ao enoturismo. Ainda nesse tópico, a grande massa de 
dados armazenada pode auxiliar as associações de produtores brasileiros em suas propostas para a obtenção de certificações geográficas - Indicação de Procedência ou Denominação de Origem.

O trabalho deixa campo aberto para pesquisas complementares. Alguns tópicos que merecem aprofundamento são: qualidade do serviço e segurança dos dados; fornecimento de serviços complementares, inclusive de maior granularidade, mais adequados a requisitos de negócio; pesquisa de soluções mais eficazes para tráfego de maiores volumes de dados (gerados por certos serviços); validação contínua do modelo produtivo do vinho. Finalmente, o modelo de utilização coletiva necessita do endosso da comunidade de produtores vinícolas brasileiros, embora o sistema possa facilmente ser modificado para utilização individual.

\section{AGRADECIMENTOS}

À Fundação de Amparo à Pesquisa do Estado de São Paulo (Fapesp), pelo apoio por meio de Auxílio à Pesquisa 06/59346-0; às vinícolas Miolo, Aurora, Salton e Aliança; ao Instituto Brasileiro do Vinho (Ibravin); à Associação dos Produtores de Vinhos Finos do Vale dos Vinhedos (Aprovale) e à Embrapa Uva e Vinho, pela colaboração neste trabalho.

\section{REFERÊNCIAS}

ABNT. ASSOCIAÇÃO BRASILEIRA DE NORMAS TÉCNICAS. NBR ISO 22000: Sistemas de gestão da segurança de alimentos - requisitos para qualquer organização na cadeia produtiva de alimentos. Rio de Janeiro, 2006. 35 p.

BECHINI, A.; CIMINO, M.G.C.A.; MARCELLONI, F.; TOMASI, A. Patterns and technologies for enabling supply chain traceability through collaborative e-business. Information and Software Technology, v.50, n. p.342-359, 2008.

BOEHM, B.W. A spiral model of software development and enhancement. Computer, Los Alamitos, v.21, n.5, p.61-72, 1988.

CONSORZIO DEL VINO BRUNELLO DI MONTALCINO. Consorzio Del Vino Brunello Di Montalcino. Montalcino: Disponível em: <http://www.consorziobrunellodimontalcino.it>. Acesso em: $1^{\underline{0}}$ set. 2006.

GOGLIANO SOBRINHO, O.; CUGNASCA, C.E. Rastreabilidade na cadeia produtiva do vinho brasileiro: 23-abr-2007. Revista Brasileira de Agroinformática - RBIAgro, São Paulo, v.7, n.1, p.44$57,2005$.

HENLEY, M.; KEMP, R. Open source software: an introduction. Computer Law \& Security Report, London, v.24, n.1, p.77-85, 2008.

JARDIM-GONÇALVES, R.; GRILO, A.; STEIGER-GARCAO, A. Challenging the interoperability between computers in industry with MDA and SOA. Computers in Industry, Groningen, v.57, n.8-9, p.679-689, 2006.

MCEACHERN, M.G.; SEAMAN, C. Consumer perceptions of meat production. British Food Journal, Bingley, v.107, n.8, p.572-593, 2005.

NATALE, O.R.; GLIELMO, L.; VASCA, F. Data modeling for batch processes data with application to winemaking. In: IEEE Conference on Decision and Control, 4., 2002, Las Vegas. Proceedings. Las Vegas: IEEE Computer Society, 2002. 6 p.

PARLAMENTO EUROPEU E CONSELHO DA UNIÃO EUROPEIA Regulamento (Ce) No 178/2002: $1^{\underline{0}}$ de fevereiro de 2002. Jornal Oficial das Comunidades Europeias, 2002. 24 p.

ROMBALDI, C.V.; TIBOLA, C.S.; FACHINELLO, J.C.; SILVA, J.A. Percepção de consumidores do Rio Grande do Sul em relação a quesitos de qualidade em frutas. Revista Brasileira de Fruticultura, Jaboticabal, v. 29, n. 3, p. 681-684, 2007. 
WORLD WIDE WEB CONSORTIUM. Web Services Glossary. Cambridge: Disponível em: <http://www.w3.org.TR/ws-gloss/>. Acesso em: 1ํago. 2007.

WORLD WIDE WEB CONSORTIUM. SOAP Specifications. Cambridge: 2007. Site Internet da especificação do protocolo SOAP, mantido pelo W3C. Disponível em: <http://www.w3.org/TR/soap/>. Acesso em: $1^{0}$ ago. 2007. 